\title{
Validation of quantitative analysis of intravascular ultrasound images
}

\author{
L. Wenguang, ${ }^{1}$ W.J. Gussenhoven, ${ }^{1,4}$ Y. Zhong, ${ }^{1}$ S.H.K. The, ${ }^{1,4}$ C. Di Mario, ${ }^{*}$ S. Madretsma, ${ }^{1}$ \\ F. v. Egmond, ${ }^{1}$ P. d. Feyter, ${ }^{1}$ H. Pieterman, ${ }^{2}$ H. v. Urk,${ }^{3}$ H. Rijsterborgh ${ }^{1,4}$ \& N. Bom ${ }^{1,4}$ \\ ${ }^{1}$ Thoraxcenter, Departments of ${ }^{2}$ Radiology and ${ }^{3}$ Vascular Surgery, University Hospital Rotterdam-Dijkzigt, \\ Erasmus University, Rotterdam and ${ }^{4}$ The Interuniversity Cardiology Institute, The Netherlands
}

Key words: intravascular ultrasound, validation, quantification

\begin{abstract}
This study investigated the accuracy and reproducibility of a computer-aided method for quantification of intravascular ultrasound. The computer analysis system was developed on an IBM compatible PC/AT equipped with a framegrabber. The quantitative assessment of lumen area, lesion area and percent area obstruction was performed by tracing the boundaries of the free lumen and original lumen.

Accuracy of the analysis system was tested in a phantom study. Echographic measurements of lumen and lesion area derived from 16 arterial specimens were compared with data obtained by histology. The differences in lesion area measurements between histology and ultrasound were minimal (mean \pm SD: $-0.27 \pm$ $\left.1.79 \mathrm{~mm}^{2}, \mathrm{p}>0.05\right)$. Lumen area measurements from histology were significantly smaller than those with ultrasound due to mechanical deformation of histologic specimens $\left(-5.38 \pm 5.09 \mathrm{~mm}^{2}, \mathrm{p}<0.05\right)$. For comparison with angiography, 18 ultrasound cross-sections were obtained in vivo from 8 healthy peripheral arteries. Luminal areas obtained by angiography were similar to those by ultrasound $\left(-0.52 \pm 5.15 \mathrm{~mm}^{2}, \mathrm{p}>\right.$ $0.05)$. Finally, intra- and interobserver variability of our quantitative method was evaluated in measurements of 100 in vivo ultrasound images. The results showed that variations in lumen area measurements were low $(5 \%)$ whereas variations in lesion area and percent area obstruction were relatively high $(13 \%, 10 \%$, respectively).

Results of this study indicate that our quantitative method provides accurate and reproducible measurements of lumen and lesion area. Thus, intravascular ultrasound can be used for clinical investigation, including assessment of vascular stenosis and evaluation of therapeutic intervention.
\end{abstract}

\section{Introduction}

Intravascular ultrasound imaging is a new technique providing real-time, cross-sectional, high resolution images of the arterial wall. The capability to obtain absolute measurements of arterial cross-sectional dimensions, such as lumen area and lesion thickness, makes this tomographic technique more suitable for quantitative assessment of the extent and severity of arterial atherosclerotic diseases [1-2]. Several studies have demonstrated the feasibility of intravascular ultrasound in qualitative as well as quantitative assessment of vascular stenosis, and its potential clinical application to evaluate intravascular intervention [3-5]. However, extensive study is still necessary to determine the adequacy of quantitative analysis regarding observer variations in measurements and possible error sources in the imaging mechanism. A computer analysis system has been developed in our lab-

* Dr. C.D. Mario, Div. of Cardiology, Vicenza, Italy, is the recipient of the E.S.C. Research Fellowship 1991. 


\section{Configuration}

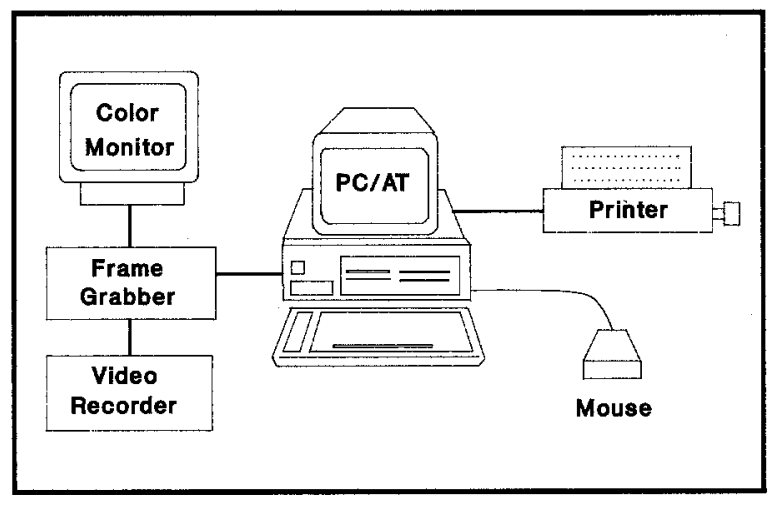

Fig. 1. Configuration of the computer analysis system.

oratory to provide quantitative analysis of intravascular ultrasound images. The purpose of this study was to validate the accuracy and reproducibility of the computerized method for quantification of intravascular ultrasound through in vitro and in vivo studies of both normal and diseased arteries.

Four steps were followed: 1) accuracy of the analysis system was evaluated in a phantom study; 2) lumen and lesion area measurements from in vitro echograms of arterial specimens were compared with those from histology; 3) lumen areas obtained from in vivo studies of normal peripheral arteries were compared with data from standard angiography; 4) intra- and interobserver variability in measurements of lumen area, lesion area and percent area obstruction was studied using images from current clinical trials of intravascular ultrasound in diseased peripheral arteries.

\section{Methods}

\section{Intravascular ultrasound system}

The intravascular ultrasound imaging system (DuMed, Rotterdam, The Netherlands) comprises a $32 \mathrm{MHz}$ single-element transducer mounted on the tip of a $5 \mathrm{~F}$ catheter and rotated by a flexible driveshaft. Cross-sectional images with $512 \times 512$ pixels and 256 grey levels can be scanned at a maximum speed of 16 frames per second. Axial resolution of the system is $<0.1 \mathrm{~mm}$. The maximum radiums scan depth is $9 \mathrm{~mm}$. Acquired physiological signals such as ECG and blood pressure can be displayed on the screen simultaneously.

\section{Computer methodology}

The computer analysis system has been described previously [6]. Briefly, the analysis system was developed on an IBM compatible PC/AT equipped with a DT 2851 framegrabber and a PC mouse for manual contour tracing (Fig. 1). The analysis procedure consists of three main steps: image acquisition, contour tracing and parameter calculation. During image acquisition, video signals from a standard VHS videotape are converted into $512 \times$ $512 \times 8$ bits digital image data with the framegrabber and stored on the hard disk of the PC. Manual tracings of the circumferential outline are processed by the computer to produce a smoothed, connected and closed contour. Parameter calculations are automatically performed after the contour has been completed or modified.

\section{Boundary tracing}

The contour of the free lumen was obtained by tracking the bright echoes of the internal elastic lamina. In the presence of a lesion, the inner boundary of the lesion was traced. For in vivo images containing strong backscatter echoes from flowing blood, the real-time images were reviewed several times on a separate videomonitor to identify blood backscatter echoes, which appeared as spontaneous contrast within the lumen.

To obtain the measurement of lesion area, the contour of the original lumen was derived from the typical three-layered appearance of a muscular vessel [7-8]. The original luminal contour overlapped the free luminal contour in the region with a normal arterial wall. For the region containing a lesion, the interface between the lesion and the underlying echolucent media was traced to estimate the boundary of the original lumen.

With the traced contours of the free and original lumen, the lesion area is simply defined as the region enclosed by these two contours. An example of tracing the free and original luminal contours is given in Fig. 2. 


\section{Parameter calculation}

Both free and original luminal areas were computed by counting the pixels enclosed by these contours. The lesion area was derived by subtracting the area of the free lumen from that of the original lumen. A mean diameter $(\mathrm{d}=2 \mathrm{r})$ of the free lumen was defined using a circular model $\left(A=\pi r^{2}\right)$. The percent area obstruction was derived by the following equation:

$$
\begin{aligned}
& \text { Percent area obstruction }= \\
& \frac{\text { (original lumen area }- \text { free lumen area) }}{\text { original lumen area }} \times 100
\end{aligned}
$$

Calibration was performed by measuring the scale markers on the images both vertically and horizontally and converting the pixels into square millimeters.

\section{Phantom study}

To test the accuracy of the analysis system, three phantoms containing water-filled cylinders with diameters of 4,8 and $16 \mathrm{~mm}$ were used. The catheter was positioned coaxially at the center of the cylinders. Each of the phantoms was imaged five times by the ultrasound system and measured using the computer analysis system described above.

In vitro measurements compared with histology Specimens obtained from 16 peripheral arteries were embedded in $1.2 \%$ agar-agar solution and filled with purified water. The catheter was placed at the center of the specimen and a number of crosssections were obtained from proximal to distal at $1 \mathrm{~mm}$ intervals. The echogram showing the most significant lesion was selected for comparative purposes. Lumen and lesion areas were measured and compared with the corresponding histologic data.

The histologic sections were analyzed using a commercially available system (IBAS, Kontron, U.S.A.). Lumen and lesion areas were calculated from manual tracings of the edges of the lumen and the internal elastic lamina.

In vivo studies compared with angiography

For comparison with angiography, 18 in vivo echo

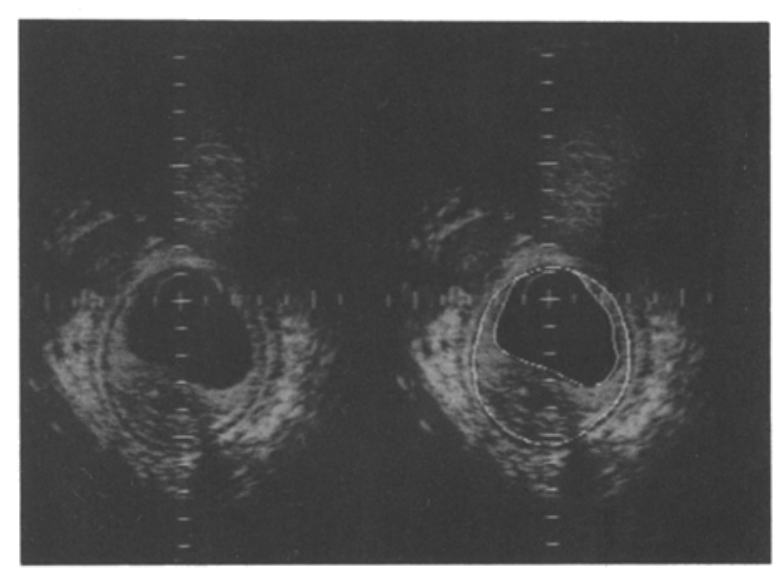

Fig. 2. Intravascular ultrasound of the superficial femoral artery (left) and the traced contours of the free and original lumen (right). The region enclosed by the two contours is the lesion.

images were obtained from 8 normal peripheral arteries. For each of 18 arterial cross-sections, echo images of early-systole and end-diastole were analyzed separately. Mean lumen area was calculated from these two measurements and used for the comparison.

Cinefilms acquired at the same arterial position were measured using the Coronary Angiography Analysis System [9]. The angiographic measurement of the lumen area was estimated with the measured diameter based on a circular model.

\section{Intra- and interobserver variability study}

Intra- and interobserver variability was studied in measurements of 100 in vivo intravascular ultrasound images obtained from 22 patients who underwent balloon angioplasty of the superficial femoral artery.

The study was performed by two independent observers. Both observers were instructed to review the real-time echo images during the contour tracing. One of the observers repeated the measurements one month later. The observer variations were analyzed in quantitative assessments of lumen area, lesion area and percent area obstruction.

\section{Statistical analysis}

Agreement between the two techniques was assessed by calculating the mean values and standard 

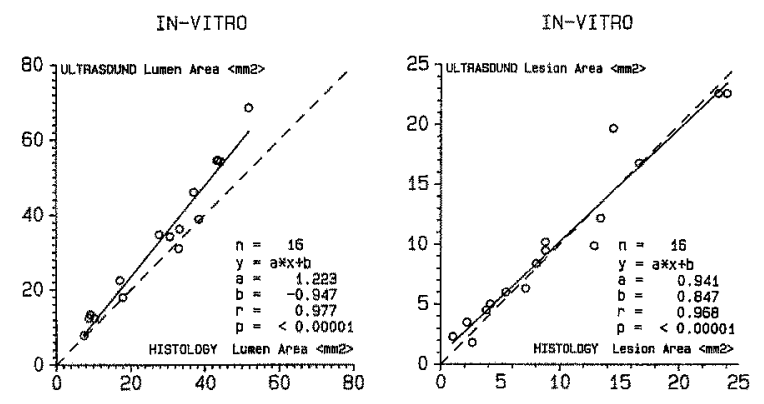

Fig. 3. Comparison of histology and ultrasound data for lumen area (left) and lesion area (right).

deviations of the paired differences [10]. Linear regression with the method of least squares was used to calculate the slopes and intercept parameters.

Observer differences were assessed with the mean values and standard deviations of the paired differences. The degree of observer variability was estimated by standard deviations of the paired differences.

The significance of the differences for data was determined by a paired t-test. A probability value of $<0.05$ was considered significant.

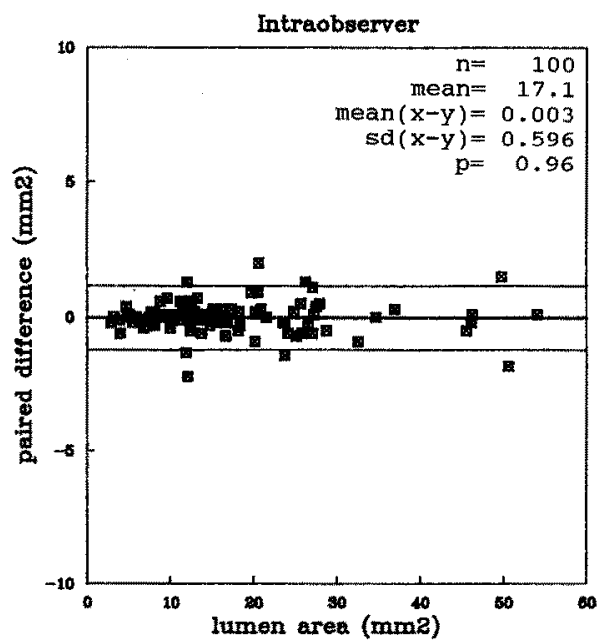

IN-VIVO

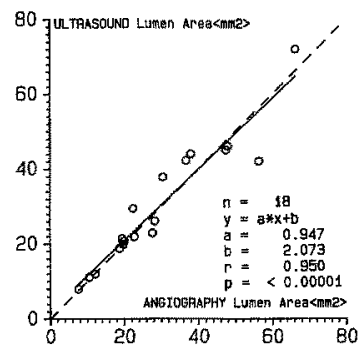

Fig. 4. Comparison of angiography and ultrasound data for hmen area.

\section{Results}

\section{Phantom study}

Accuracy of the analysis system for the phantom measurements was very high (mean diameter $\pm \mathrm{SD}: 3.84 \pm 0.01(4 \mathrm{~mm}), 7.97 \pm 0.04(8 \mathrm{~mm})$, and $16.24 \pm 0.06(16 \mathrm{~mm})$, respectively). The data showed excellent agreement with the dimensions of the phantoms.

\section{Comparison with histology}

The regression equations for data between histology and ultrasound were $\mathrm{y}=1.22 \times$ [histology] 0.95 for lumen area and $y=0.94 \times[$ histology $]+$ 0.85 for lesion area (Fig. 3). No significant differences in lesion area measurements were noted be-

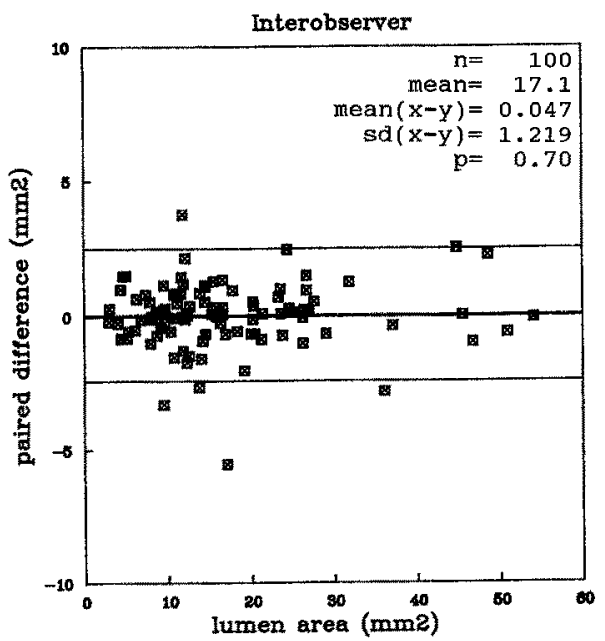

Fig. 5. Intra- and interobserver variability in lumen area measurements. The $95 \%$ confidence interval is indicated by the upper (mean + $2 \mathrm{SD}$ ) and lower (mean - 2SD) vertical lines. 

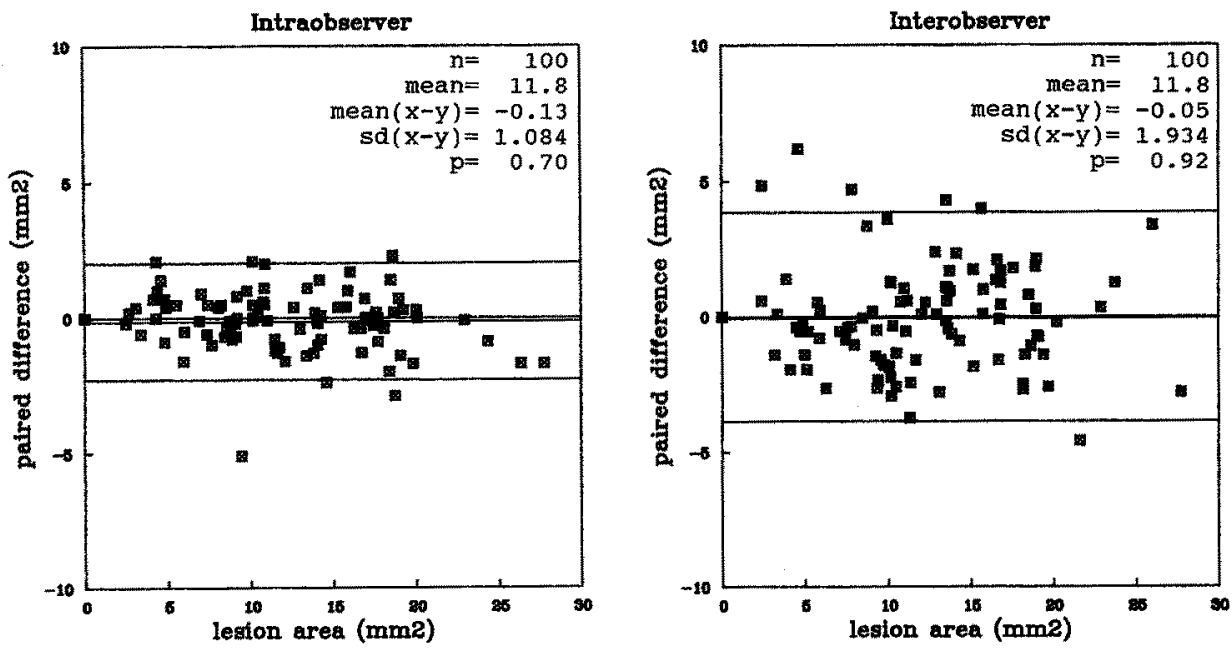

Fig. 6. Intra- and interobserver variability in lesion area measurements. The $95 \%$ confidence interval is indicated by the upper (mean + 2SD) and lower (mean - 2SD) vertical lines.

tween the two methods $\left(-0.27 \pm 1.79 \mathrm{~mm}^{2}, \mathrm{p}>\right.$ $0.05)$. However, the mean lumen area obtained by histology was $17 \%$ smaller than with ultrasound $\left(-5.38 \pm 5.09 \mathrm{~mm}^{2}, \mathrm{p}<0.05\right.$, mean of the measurements: $31.1 \mathrm{~mm}^{2}$ ).

\section{Comparison with angiography}

The regression result of lumen area measurements from angiography and ultrasound was close to the line of identity $(y=0.95 \times$ [angiography] +2.07$)$ (Fig. 4). Lumen area measurements obtained by

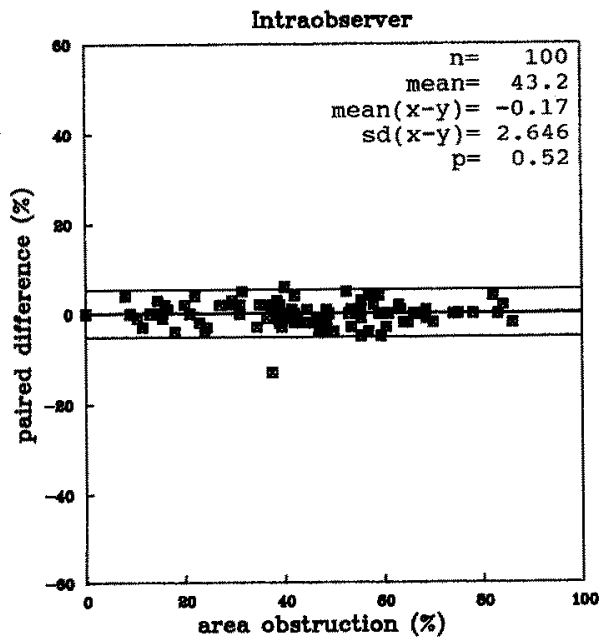

ultrasound were similar to those with angiography $\left(-0.52 \pm 5.15 \mathrm{~mm}^{2}, \mathrm{p}>0.05\right)$

Intra- and interobserver variability

Data of observer variability studies in measurements of lumen area, lesion area and percent area obstruction are shown in Figs 5-7, with scatter plots of the differences versus the measured mean values. For all measurements, no significant observer bias (a non-zero mean of the paired differences) was present. In both intra- and interobserver dif-

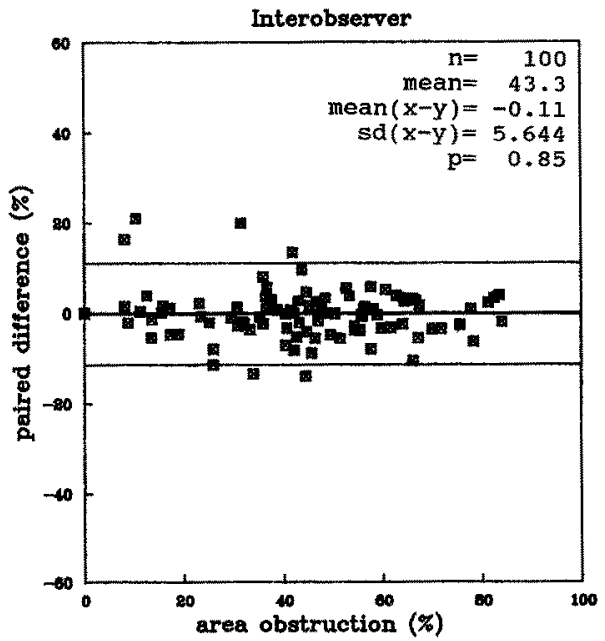

Fig. 7. Intra- and interobserver variability in percent area obstruction measurements. The $95 \%$ confidence interval is indicated by the upper (mean $+2 S D)$ and lower (mean $-2 S D)$ vertical lines. 
ferences, observer variations (standard deviations of the paired differences) in lumen area measurements were relatively smaller than those of lesion area measurements (intraobserver: $0.6 \mathrm{~mm}^{2}$ for $\mathrm{lu}$ men area vs $1.1 \mathrm{~mm}^{2}$ for lesion area, interobserver: $1.2 \mathrm{~mm}^{2}$ for lumen area vs $1.9 \mathrm{~mm}^{2}$ for lesion area). Intra- and interobserver variations in measurements of percent area obstruction were $2.6 \%$ and $5.6 \%$, respectively.

\section{Discussion}

In both in vitro and in vivo studies, quantitative assessment of intravascular ultrasound agreed well with histology and angiography except for the $17 \%$ underestimate in histologic lumen area measurements. The underestimate by histology can probably be attributed to the fixation and tissue processing during histologic preparation of the specimens. After this procedure, the shape of the vessel lumen may alter and tissue shrinkage occur, resulting in a slight decrease in histologic measurements. For comparison with angiography, it should be noted that intravascular ultrasound is a tomographic technique and angiography is a silhouette technique. Thus, identical results may only be expected in vessels with circular geometry. For healthy vessels with almost circular cross-sections, no significant differences were found in our comparison study with angiography.

The images used in the observer variability studies were of clinical quality, selected by another clin ical group for assessment of the effect of vascular intervention with intravascular ultrasound. The aim was to evaluate observer variability under conditions similar to practical applications. For a rough guide of observer variability from our results, one may estimate observer variations in percent with standard deviations of the paired differences in relation to their mean measurements. Using the means of intra- and interobserver data, the percent observer variations for quantitative data of lumen area, lesion area and percent area obstruction were $5 \%, 13 \%$ and $10 \%$, respectively. The repeatability in the quantitative assessment of lumen area was very good. The influence of blood backscatter echoes on determination of the luminal contour can be diminished by reviewing the realtime images. The higher degree of observer variations in lesion area measurements suggests that it is relatively more difficult to determine the boundary of the media than the arterial lumen. This is intimately related to the presence of an advanced atherosclerotic lesion with partial dropout of far-field echoes, and complex wall structure such as rupture or dissection of the lesion following intervention.

Other factors may also affect the accuracy of quantitative results, including minor differences in sound velocity between saline and blood [11], graphic distortion caused by the mechanical system [12] and overestimation of the cross-sectional dimensions due to the off-axis catheter position [13]. Of these three factors, the off-axis catheter position seems to be the most serious problem. Although the angle between the catheter and the arterial wall was difficult to determine for in vivo measurements, our experience in peripheral artery studies showed that, in most cases, the catheter was aligned with the normal or minimal diseased part of the wall. With straighter peripheral arteries one can expect that alignment allows an approximately co-axis catheter position.

\section{Conclusion}

In the phantom study and the comparison with histology and angiography, we demonstrated the feasibility to obtain accurate quantitative assessment of lumen, lesion area and percent area obstruction. Observer variability in measurements proved to be satisfactorily low in terms of analyzing images from a new technique. Using our method, quantitative analysis of intravascular ultrasound can be used for clinical applications such as assessment of vascular stenosis and evaluation of therapeutic intervention.

\section{References}

1. Bom $\mathrm{N}$, Hoff $\mathrm{H}$ ten, Lancée CT, Gussenhoven WJ, Bosch JG. Early and recent intraluminal ultrasound devices. Int $\mathbf{J}$ Card Imaging 1989; 4: 79-88. 
2. Roelandt $\mathbf{J}$, Serruys $\mathbf{P W}$. Intraluminal real-time ultrasonic imaging: Clinical perspectives. Int J Card Imaging 1989; 4: 89-97.

3. Pandian NG, Weintraub A, Kreis A, Schwartz SL, Konstam MA, Salem DN. Intracardiac, intravascular, two dimensional high frequency ultrasound imaging of pulmonary artery and its branches in humans and animals. Circulation 1990; 81: 2007-12.

4. Neville RF, Bartorelli AL, Sidawy AN, Almagor Y, Potkin $\mathrm{B}$, Leon MB. An in vivo feasibility study of intravascular ultrasound imaging. Am J Surg 1989; 158: 142-5.

5. Tobis JM, Mallery JA, Gessert J, Griffith J, Mahon D, Bessen M, Moriuchi M, McRae M, Henry WL. Intravascular ultrasound cross-sectional arterial imaging before and after balloon angioplasty in vitro. Circulation 1989; 80: 873-82.

6. Wenguang L, Gussenhoven WJ, Bosch JG, Mastik F, Reiber JHC, Bom N. A computer-aided analysis system for the quantitative assessment of intravascular ultrasound images. Computers in Cardiology 1990: 333-6.

7. Gussenhoven EJ, Essed CE, Lancée CT, Mastik F, Frietman P, Egmond F van, Reiber J, Bosch H, Urk Hv, Roelandt $\int$, Bom $N$. Arterial wall characteristics determined by intravascular ultrasound imaging: An in vitro study. J Am Coll Cardiol 1989; 14: 947-52.

8. Potkin BN, Bartorelli AL, Gessert JM, Neville RF, Almagor $\mathrm{Y}$, Roberts WC, Leon MB. Coronary artery imaging with intravascular ultrasound. Circulation 1990: 81: 1575-85.
9. Reiber JHC, Serruys PW, Kooijman CJ. Assessment of short-, medium-, and long-term variations in arterial dimensions from computer-assisted quantitation of coronary cineangiograms. Circulation 1985; 71: 280-8.

10. Bland JM, Altman DG. Statistical methods for assessing agreement between two methods of clinical measurement. Lancet 1986; February 8: 307-10.

11. Moriuchi M, Tobis JM, Mahon D, Gessert J, Griffith J, McRae M, Moussabeck O, Henry WL. The reproducibility of intravascular ultrasound imaging in vitro. J Am Soc Echo 1990; 3: 444-50.

12. Hoff $H$ ten, Korbijn A, Smit TH, Klinkhamer JFF, Bom N. Imaging artifacts in mechanically driven ultrasound catheters. Int J Card Imaging 1989; 4: 195-9.

13. Nishimura RC, Edwards WD, Warnes CA, Reeder GS, Holmes DR, Tajik AJ, Yock PG. Intravascular ultrasound imaging: In vitro validation and pathologic correlation. $J$ Am Coll Cardiol 1990; 16: 145-54.

Address for offprints:

L. Wenguang,

Thoraxcenter (Ee 2302),

Erasmus University,

P.O. Box 1738,

3000 DR Rotterdam,

The Netherlands 